This is the final peer-reviewed accepted manuscript of:

C.I. Kourogiorgas et al

"Capacity Statistics Evaluation for Next Generation Broadband MEO Satellite Systems"

in: IEEE Transactions on Aerospace and Electronic Systems, vol. 53, no. 5, pp. 2344-2358, Oct. 2017

The final published version is available online at:

https://doi.org/10.1109/TAES.2017.2693018

Rights / License:

The terms and conditions for the reuse of this version of the manuscript are specified in the publishing policy. For all terms of use and more information see the publisher's website.

This item was downloaded from IRIS Università di Bologna (https://cris.unibo.it/)

When citing, please refer to the published version. 


\section{Capacity Statistics Evaluation for Next Generation Broadband MEO Satellite Systems}

Charilaos I. Kourogiorgas, Member, IEEE, Daniele Tarchi, Senior Member, IEEE, Alessandro Ugolini, Nikolaos Lyras, Pantelis-Daniel Arapoglou, Athanasios D. Panagopoulos, Senior Member, IEEE, Giulio Colavolpe, Senior Member, IEEE, Alessandro Vanelli-Coralli, Senior Member, IEEE

\begin{abstract}
In this paper, the performance of a reference Medium Earth Orbit (MEO) satellite constellation system operating at Ka-band and employing single links to ground is compared with next generation advanced systems in higher RF or optical bands employing multiple diversity links. The fill rate of existing MEO constellations offering broadband and trunking services in Ka-band is growing fast rendering the search for additional spectrum of vital importance. Therefore, this paper reports on the results of a system study investigating the option of using Q/V band, or even optical wavelengths, instead of Ka-band, to deliver substantially higher system capacity. The system study takes a holistic approach covering from atmospheric channel impairments to waveform optimization and system analysis for realistic assumptions. After proposing a sophisticated channel model to generate spatio-temporal time series of atmospheric attenuation, an optimization of the performance at physical layer is performed to derive the inputs necessary to the system analysis. Five different advanced high frequency RF and optical systems are compared in terms of outage capacity and availability for various locations of single ground stations, multiple ground stations (site diversity) and from multiple satellites (orbital diversity). For maximizing the realism of the comparison, similar on board satellite resources (mass, power) are assumed for all scenarios.
\end{abstract}

Index Terms-MEO constellation; Trunking system; Outage Capacity; Space-Time synthesizer;

\section{INTRODUCTION}

Although, most satellite communication systems are employing Low Earth Orbit (LEO) or Geostationary Earth Orbit (GEO) satellites, very recently the use of Medium Earth Orbit (MEO) satellites has shown great potential and has led to a high capacity fill rate of the multiple satellites belonging to the constellation [1]. In order to provide quasi-global services a smaller number of MEO satellites is needed compared to a LEO constellation. Moreover, free space losses and the latency due to radiowave propagation is smaller than in GEO links [2].

This work was supported by SatNEX-IV program of European Space Agency.

C. I. Kourogiorgas, N. Lyras, A. D. Panagopoulos are with School of Electrical and Computer Engineering, National Technical University of Athens, Iroon Polytechniou 9, Zografou- Athens (E-mail: harkour@mail.ntua.gr, thpanag@ece.ntua.gr). D. Tarchi and A. Vanelli Coralli are with the Departiment of Electrical, Electronic and Information Engineering, University of Bologna, Italy (E-mail: daniele.tarchi@unibo.it, alessandro.vanelli@unibo.it) A. Ugolini and G. Colavolpe are with Dipartimento di Ingegneria e Architettura, University of Parma, Parco Area delle Scienze 181/A, I-43124 Parma, Italy (E-mail: alessandro.ugolini@unipr.it, giulio.colavolpe@unipr.it). P.-D. Arapoglou is with European Space Agency (ESA) / European Space Research \& Technology Centre (ESTEC), Keplerlaan 1, 2201 AZ Noordwijk, The Netherlands (E-mail: pantelis-daniel.arapoglou@esa.int).
The O3b system is already operational in Ka-band and uses ten satellites in equatorial orbit [1], while other MEO systems in similar orbits are currently under investigation, even at optical wavelengths [3].

Owing to this high demand for broadband and trunking traffic from MEO, it is of interest to pursue technologically advanced solutions that can offer additional spectrum for next generation systems. One step-wise option is to employ bands higher than $\mathrm{Ka}(20 / 30 \mathrm{GHz})$, namely Q/V-band $(40 / 50 \mathrm{GHz})$. A more disruptive approach is to consider communication technology at optical wavelengths. In Q/V band, atmospheric phenomena are harsher than in Ka-band. In particular, rain, atmospheric gases and clouds cause attenuation of the signal, while amplitude scintillation occurs due to the turbulence in the troposphere [4]. Although, rain is the dominant fading mechanism at both Q- and Ka-bands, the atmospheric attenuation is increased compared to the attenuation at Ka-band. Therefore, the use of Fade Mitigation Techniques (FMTs) [4] must be considered.

Adaptive Coding and Modulation (ACM) is a FMT which has been already adopted in the second generation of ETSI standard for Digital Video Broadcasting on satellite links, DVB-S2 [5] and its extension DVB-S2X [6]. DVB-S2 has already been studied for the High Throughput Satellite systems [7] and it is also implemented in the currently operational $\mathrm{O} 3 \mathrm{~b}$ system. However, at Q/V-band, ACM may not be enough to achieve the desired link availability due to atmospheric attenuation and, therefore, spatial diversity becomes necessary. Two spatial diversity architectures are of interest: a) Site diversity: in which two or more ground stations are communicating with the same satellite and b) Orbital diversity: one ground station communicates with two or more satellites. In both techniques, the links that are used for the communication between Earth and satellite are spatially separated. This spatial separation decreases the correlation of atmospheric attenuation induced in the links. The performance of a MEO constellation for single links at Ka and Q-bands has been also recently evaluated using ACM technique for a small number of ground stations at [8] and [9], respectively.

This paper reports on a system study performed under the SatNEx IV framework [10] comparing various options in next generation MEO satellite systems. Having as reference a system similar to today's O3b operating at Ka-band, we investigate the benefits in terms of system capacity (equivalent 
throughput) of migrating to Q-band. ${ }^{1}$ Being a more disruptive approach, we also devote a smaller part of the system study to a MEO system option employing optical wavelengths. In total, five systems with different operating frequencies and spatial techniques are considered: a) single link Ka-band, b) single link Q-band, c) site diversity at Q-band, d) orbital diversity at Q-band and e) optical wavelength. The comparison among these five system options is done to the extent possible based on equal or similar mass and power satellite payload resources.

The system study follows a holistic approach covering all necessary elements: a) the simulation of the orbit for the whole MEO constellation, b) providing an accurate spatio-temporal time series synthesizer as an atmospheric channel model c) an in depth waveform and physical layer optimization using a realistic satellite payload model. All this is done to finally run the system level simulations using the outputs of these modeling efforts. The sophisticated channel model is developed in order to generate time series of attenuation for single link and spatially separated links reproducing the spatial and temporal behavior of atmospheric attenuation. Furthermore, being a broadband interactive system, the RF system options assume ACM employing a wide range of possible modulation and coding (MODCOD) options from the DVB-S2 and DVBS2X standards [5], [6]. The thresholds for these MODCODs are calculated based on thorough optimization of the physical layer.

The remainder of the paper is organized as follows: in Section II, the system, orbital and geometric characteristics are given. In Section III, the channel model used for the generation of the atmospheric attenuation time series is described, while in Section IV, the optimization methodology on the physical layer for the calculation of the MODCOD tables is presented. In Section V, the simulation methodology, as well as numerical results on the capacity statistics are presented.

\section{MEO SYSTEM DEFINITION AND CHARACTERISTICS}

In order to fairly compare the various system options, a number of common system limitations are adopted. Firstly, it is assumed that the DC power consumption on-board the satellite payload must be equal to $1300 \mathrm{~W}$, the system must at least provide a target availability of $99.9 \%$ and the total acceptable propagation latency constraint for the complete forward link (gateway, satellite, user terminal) has been set to $150 \mathrm{~ms}$. The latter value is indicative of delay intolerant services, which is a MEO stronghold. Finally, as the Ka- and Q-band systems use both the same antenna sizes and the same frequency re-use across their spot beams, the mass of the satellite payload also turns out similar.

It is found from [11] that the frequency interval between $37.5 \mathrm{GHz}$ and $42.5 \mathrm{GHz}$ belonging to the fixed satellite service could be used, even if it is also allocated to highdensity applications. Therefore, for every satellite an available bandwidth of $5 \mathrm{GHz}$ is assumed.

The constellation considered in our study consists of $8 \mathrm{MEO}$ satellites with equal spacing, i.e. $45^{\circ}$. The satellite altitude is

\footnotetext{
${ }^{1}$ As our study is limited to the satellite to user terminal downlink, we only refer to Q-band in the following.
}

TABLE I: Chosen Ground Stations

\begin{tabular}{ccc}
\hline Location & Latitude (deg) & Longitude (deg) \\
\hline \hline Nemea, Greece & $37.8^{\circ}$ & $22.65^{\circ}$ \\
Lima, Peru & $-12.04^{\circ}$ & $-77.04^{\circ}$ \\
Hawaii, US & $19.74^{\circ}$ & $-155.65^{\circ}$ \\
Dubbo, Australia & $-32.25^{\circ}$ & $148.61^{\circ}$ \\
Vernon, Texas, US & $34.15^{\circ}$ & $-99.30^{\circ}$ \\
Karachi, Pakistan & $24.96^{\circ}$ & $67.06^{\circ}$ \\
Sintra, Portugal & $38.8^{\circ}$ & $-9.38^{\circ}$ \\
Hortolandia, Brazil & $-22.97^{\circ}$ & $-47.21^{\circ}$ \\
\hline
\end{tabular}

$8062 \mathrm{~km}$ above mean sea level and the inclination angle is less than $0.1^{\circ}$. Each satellite has 12 steerable antennas, 10 of which are used for the user beams while 2 of them are used for the GWs. The antenna diameter is approximately $22 \mathrm{~cm}$. Each steerable antenna creates a single spot beam on ground and is fed by a unique transponder. As mentioned, between the Ka- and Q-band systems we assume the same frequency re-use factor equal to two. Reusing the available in Q-band $5 \mathrm{GHz}$ twice per satellite, each transponder has 833 $\mathrm{MHz}$ of bandwidth available. The term transponder should be understood here as a payload chain with a single Traveling Wave Tube Amplifier (TWTA) with a saturated RF power of $65 \mathrm{~W}$ with a $50 \%$ efficiency.

To capture the variability of climatic conditions and corresponding propagation effects, eight (8) ground station locations are assumed for the user links. The selected stations as well as their locations are shown in Table I. ${ }^{2}$ Two classes of UT antennas will be used: $1.8 \mathrm{~m}$ and $3.5 \mathrm{~m}$. The global picture of the MEO constellation as well as the selected ground stations are depicted in Fig. 1. In the same figure, the contour line for the $5^{\circ}$ elevation angle is shown for each satellite to highlight the latitude of the possible satellite coverage.

Moreover, it is assumed that every UT is equipped with two antennas. This is a typical operating condition in existing MEO systems as it allows for a seamless satellite handover, since one of the antennas points at the rising satellite and the other one at the descending satellite. The UT is assumed to always receive data from the satellite at the highest elevation angle.

The geometry of an orbital (satellite) diversity architecture is shown in Fig. 2. The separation angle between the two links is denoted as $\Delta \psi$ and the links may have different elevation angles toward the different satellites. Due to the fact that every UT is equipped with two antennas, orbital diversity is possible in case both rising and descending satellites are transmitting the same signal to the user. Of course, this implies that a neighboring satellite has such capacity to spare. As for the choice of which link to select, the UT is communicating using the link with the highest Signal-to-Noise Ratio (SNR) suffering from minimum attenuation.

For the site diversity system, two UTs separated by a distance $d$ communicate with a single satellite (Fig. 3). The two links are assumed to be at the same elevation angle. Therefore, each UT of the site diversity system has two antennas and the data are delivered to Earth through the

\footnotetext{
${ }^{2}$ The chosen locations are the ones also chosen by $\mathrm{O} 3 \mathrm{~b}$ for the installation of the GWs
} 


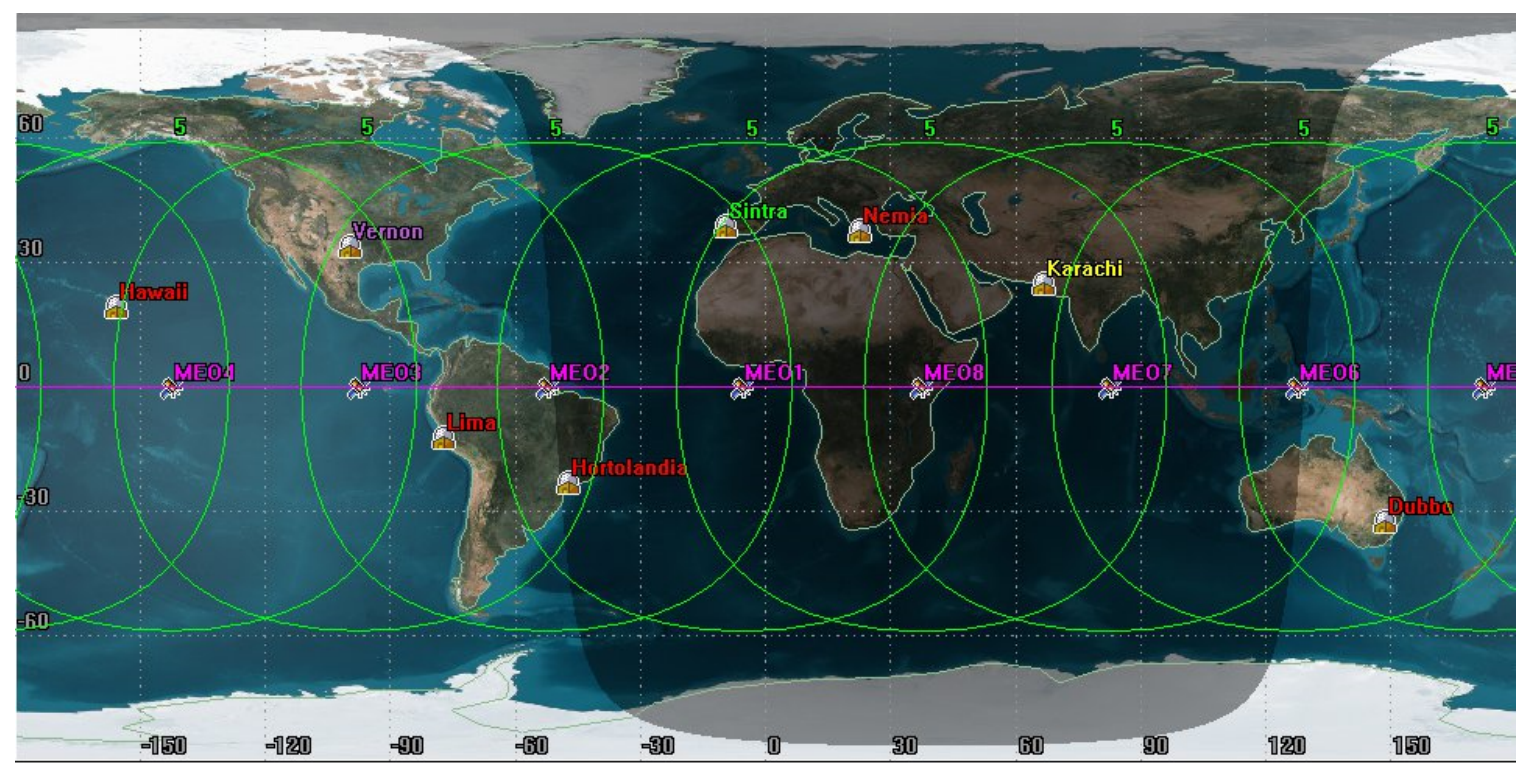

Fig. 1: MEO constellation and selected ground stations for system studies

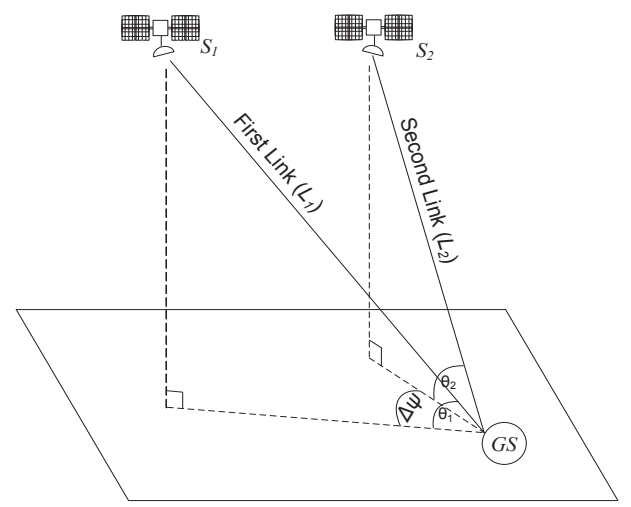

Fig. 2: System Geometry for the Orbital Diversity System

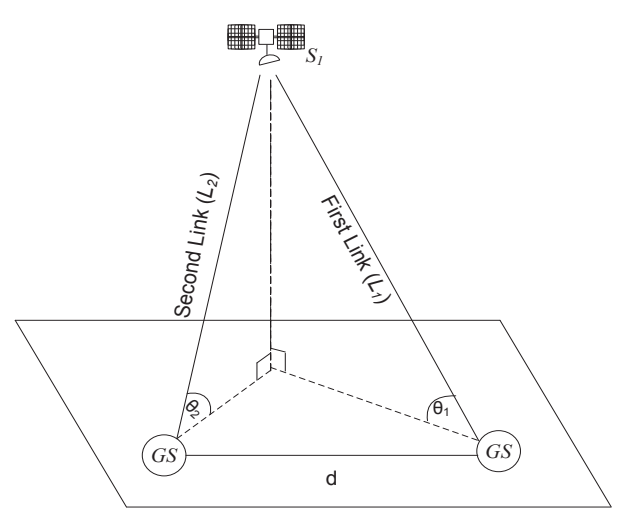

Fig. 3: System Geometry for site diversity system

satellite seen at the highest elevation angle. The UT with the least attenuation is chosen for the data delivery.

Turning next to the case of the optical MEO system, in line with the state-of-the-art, we assume that an optical terminal on board the satellite consumes $160 \mathrm{~W}$ of DC power [12]. Then, to preserve the power envelope of $1300 \mathrm{~W}$, on board each satellite 8 optical terminals are embarked. Current state-of- the-art of these terminals achieves a data rate per of about 5.6 Gbps [12]. Looking to the next generation of MEO systems in the mid-term, we extrapolate this data rate to $20 \mathrm{Gbps}$, assuming, e.g., a spectrally more efficient modulation or the possibility for wavelength division multiplexing.

\section{Channel MODEL}

In order to evaluate the system performance for diversity systems, a space-time synthesizer of atmospheric attenuation is developed, which is able to capture the spatial and temporal behavior of the medium. Total atmospheric attenuation $A_{a t m}$ time series are calculated summing up the time series of the effects of each factor [13]:

$A_{\text {atm }}(t)=A_{\text {rain }}(t)+A_{c l}(t)+A_{w v}(t)+A_{\text {oxygen }}(t)+S(t)$

where $A_{\text {rain }}, A_{c l}, A_{w v}, A_{\text {oxygen }}$ and $S$ are the rain attenuation, cloud attenuation, attenuation due to water vapor, oxygen attenuation, and scintillation, respectively, all in decibels. Here it shall be noted that, although ITU-R Recommendation P.1853 [13] provides a methodology for generating atmospheric attenuation for GEO links, it is not directly applicable to the dynamic MEO links studied in this paper.

\section{A. Rain Attenuation}

Rain attenuation is generated using the extension of methodology in proposed in [14] to take into account the timedependence of elevation angles and the correlation between the spatially separated links for orbital and site diversity. The synthesizer assumes that for a given elevation angle $(\theta)$, rain attenuation is considered as a lognormal distribution with statistical parameters $A_{m}(\theta)$ and $S_{A}(\theta)$. However, due to the MEO satellite orbital characteristics, the synthesizer considers the time dependency of $A_{m}$ and $S_{A}$ and the temporal evolution of the dynamic parameter $(\beta)$ of rain attenuation (for detailed explanation see [14]). Now, considering $n$ spatially separated 
links, rain attenuation induced on link $i$ is denoted as $A_{\text {rain, } i}$. For the generation of rain attenuation time series, the time series of the Gaussian process $\left(U_{i}\right)$ are generated for every link using the following multidimensional Stochastic Differential Equation (SDE) [15]:

$$
\mathbf{U}_{t}=e^{\int^{t} \mathbf{B}_{y} d y} \mathbf{U}_{0}+e^{\int^{t} \mathbf{B}_{y} d y} \int_{0}^{t} \exp \left(-\int_{0}^{y} \mathbf{B}_{y^{\prime}} d y^{\prime}\right) \mathbf{S}_{y} d \mathbf{W}_{y}
$$

where $\mathbf{B}_{\mathbf{t}}$ is a $n \times n$ matrix with elements $b_{i j, t}=-\beta_{i, t} \delta_{i j}$ with $\delta_{i j}$ the Kroenecker delta function and $-\beta_{i, t}$ the dynamic parameter of rain attenuation as defined in [14]. The matrix $\mathbf{B}$ is time dependent since, as shown in [14], its elements depend on the elevation angle and therefore for MEO slant paths, the dynamic parameter is time dependent. Continuing from (2), $\mathbf{W}_{\mathbf{t}}$ is the $n$-dimensional Brownian Motion, [15]. From [16], the matrix $\mathbf{S}$ is calculated from the Cholesky decomposition of matrix $\mathbf{G}_{\mathbf{U}}$ :

$$
[G]_{i j}=\left(\beta_{i}+\beta_{j}\right)\left[C_{U}\right]_{i j}
$$

where $\beta_{i}$ and $\beta_{j}$ are the dynamic parameters of rain attenuation for the links $i$ and $j$ and the $n \times n$ matrix $\mathbf{C}_{\mathbf{U}}$ is the covariance matrix of the process $U$ :

$$
\mathbf{C}_{U}=\left(\begin{array}{ccc}
1 & \cdots & \rho_{1 n} \\
\vdots & \ddots & \vdots \\
\rho_{1 n} & \cdots & 1
\end{array}\right)
$$

where $\rho_{i j}$ is the correlation between processes $U_{i}$ and $U_{j}$. Differently than for GEO, in MEO slant paths the matrix $\mathbf{S}$ is time dependent. However, there is a difference in the correlation coefficient $\rho_{i j}$ between the orbital diversity and site diversity geometries. In site diversity, the correlation coefficient for rain attenuation adopted by ITU-R. P. 618 [17] is used:

$$
\rho_{S D}=0.94 \exp (-d / 30)+0.06 \exp \left[-(d / 500)^{2}\right]
$$

where $d$ is the separation distance between the two ground stations. Therefore, in the proposed channel model the correlation coefficient of (5) for site diversity system remains constant with time. Here it must be noted that although the coefficient of (5) remains constant it does not mean that the correlation of rain attenuation does not depend on the characteristics of the links, since (5) is for the Gaussian distributed processes $U_{i}$ and $U_{j}$. However, for orbital diversity scenario a different correlation coefficient for the normalized Gaussian processes $U_{i}$ and $U_{j}$ is used which is analytically described in [18].

The rain attenuation time series on link $i$, is calculated through the following transformation:

$$
A_{i}(t)=A_{m, i}(t) \exp \left(S_{a, i}(t) U_{i}(t)\right)
$$

\section{B. Cloud Attenuation}

For cloud attenuation, the methodology recommended in ITU-R. P. 1853 [13] is extended for multiple and spatially separated links. The synthesizer for attenuation due to clouds is based on generating time series of Integrated Liquid Water Content (ILWC) on a point. Then, using the recommendation of ITU-R. P. 840 [19] the time series of attenuation due to clouds are generated from the time series of ILWC.

For the generation of ILWC time series, based on [13], the ILWC on point $i$ is calculated through:

$$
\begin{gathered}
L_{i}(t)=\exp \left(\operatorname{erfc} c^{-1}\left(\operatorname{erfc}\left(\frac{G_{C, i}(t)}{\sqrt{2}}\right) /\left(P\left(L_{i}>0\right) / 100\right)\right)\right. \\
\cdot \sqrt{2} \cdot s+m), \text { for } G_{C, i}(t)>a \\
L_{i}(t)=0 \text { for } G_{C, i}(t) \leq a
\end{gathered}
$$

where $s$ and $m$ are the statistical parameters of ILWC, $P(L>$ $0)$, the probability of cloud presence on a point derived from ITU-R. P. 840 [19]. Furthermore, $\alpha$ is calculated from:

$$
a=Q^{-1}(P(L>0))
$$

where $Q^{-1}$ is the inverse of the Gaussian-Q function. The time series of the Gaussian process $G_{C, i}$ for link $i$ are calculated from the superposition of the Gaussian time series $X_{i}^{1}$ and $X_{i}^{2}$ using:

$$
G_{C, i}\left(k T_{S}\right)=\gamma_{1} X_{i}^{1}\left(k T_{S}\right)+\gamma_{2} X_{i}^{2}\left(k T_{S}\right)
$$

where $T_{S}$ is the sampling period and the parameters $\gamma_{1}, \gamma_{2}$ are derived from [13]. The time series of $X_{i}^{1}$ and $X_{i}^{2}$ for the single link $i$ are derived through passing zero mean and unity variance white Gaussian noise from two lowpass filters with cutoff frequency $7.17 \times 10^{-4} \mathrm{~s}^{-1}$ and $2.01 \times 10^{-5} \mathrm{~s}^{-1}$, respectively. In order to extend the above mentioned methodology to multiple links, multi-dimensional correlated Gaussian noise is used. To include the spatial correlation, the $n$-dimensional noise is multiplied with a correlation matrix similar in form to (4). For the correlation coefficient the formula proposed in [20] as a function of separation distance $(d)$ is used:

$$
\rho_{C}(d)=0.35 e^{-\frac{d}{7.8}}+0.65 e^{-\frac{d}{225.3}}
$$

Therefore, in case of site diversity the correlation coefficient is constant with constant separation distance. For the orbital diversity scenario, the separation distance is set equal to the distance between the two converging links at the low cloud base, which is set equal to $1 \mathrm{~km}$ a.m.s.l. [20]. So, the correlation matrix is time dependent. Here it must be noted that, the choice of $1 \mathrm{~km}$ a.m.s.l stems from the fact that the cloud base height can be considered at this specific height [20], in order to use a constant value for the ease of calculations in this complex simulator. In order to calculate the mean of the volume causing clouds attenuation, the vertical extent of clouds must be calculated with the methodology presented in [21] which depends on the time series of ILWC.

\section{Attenuation due to Atmospheric Gases}

Attenuation also occurs due to water vapor and oxygen molecules. Similarly to cloud attenuation, the ITU-R. P. 1853 is implemented for generating time series of attenuation on a single link. However, the attenuation time series must be correlated also in the spatial domain, since spatial diversity techniques are employed.

Oxygen attenuation on a link with constant elevation angle is considered constant over time. Moreover, the same oxygen 
attenuation value is considered for the spatially separated links, due to the very high spatial correlation that oxygen distribution exhibits [22]. Therefore, attenuation due to oxygen is calculated using ITU-R. recommendation P. 676 [23] at a specific exceedance probability. The same value is used for all the spatially separated links with the same elevation angle. However, in case of MEO the oxygen attenuation changes over time, since the elevation angle of the link changes also.

The methodology for generating attenuation due to water vapor on multiple links is the extension of the methodology presented in [13]. According to ITU-R. P. 1853, firstly, the time series of Integrated Water Vapor Content (IWVC) are generated for a single point and then using ITU-R. P. 676 [23] the attenuation due to water vapor time series induced in a single link are calculated.

The IWVC on a single point $i$ is considered as a stochastic process following the Weibull distribution [24], with statistical parameters $\lambda$ and $\kappa$. The time series of IWVC on point $i$ are generated through:

$$
I W V C_{i, t}=\lambda\left(-\ln \left(Q\left(Y_{i, t}\right)\right)\right)^{1 / \kappa}
$$

where $Y_{i, t}$ are generated from lowpass filtering of zero mean unity variance white Gaussian noise. The low-pass filter is considered with a cut-off frequency of $3.24 \cdot 10^{-6} s^{-1}$. In order to extend the methodology for $n$ multiple links, $n$ low pass filters are used and the $n$-dimensional input Gaussian noise is correlated. The correlation coefficient is derived from [22]. The correlation coefficient depends on the separation distance between the links. For the case of site diversity this distance is equal to the distance between the ground stations, while for orbital diversity the separation distance is the distance between the links at a height of $1 \mathrm{~km}$ a.m.s.l.

\section{Scintillation}

The last factor that must be taken into account is the scintillation effect. Amplitude scintillation time series are generated through the modification of the methodology presented in [25]. In the latter model, stochastic differential equations driven by fractional brownian motion are used in order to generate time series following a Gaussian distribution with a lowpass power spectrum with a slope of $-80 / 3 \mathrm{~dB} / \mathrm{dec}$ ade. The methodology in [25] is modified to take into account the time dependent elevation angle. We consider scintillation between spatially separated links as uncorrelated.

\section{E. Correlation between the Attenuation Factors}

ITU-R. P. 1853 [13] takes in due account the correlation among the atmospheric constituents inducing attenuation. According to [13], the same white Gaussian noise is used to synthesize both rain attenuation and cloud attenuation. For the integrated water vapour content, a covariance equal to 0.8 between the white noise used for IWVC and the one for ILWC is introduced for every link. For scintillation, the variance is calculated according to:

$$
\sigma_{\text {scint }}=\left\{\begin{array}{c}
\sigma_{\text {sc }}, A_{\text {rain }}<1 d B \\
C A_{\text {rain }}^{5 / 12}, A_{\text {rain }} \geq 1 d B
\end{array}\right.
$$

where $\sigma_{s c}$ is derived from ITU-R. P. 618 [17] and $C$ is set equal to 0.039 and 0.056 for $\mathrm{Ka}$ - and Q-band links [26], respectively.

\section{F. Optical Links Cloud-Free-Line-of-Sight}

The liquid water particles which are present in the cloud formations are the dominant attenuation mechanisms in optical links [20], [27], [21]. Therefore, it is assumed that links are available only when a cloud-free environment exists. Thus, the availability of the system is equal to the probability that clouds are not present, i.e. Cloud-Free-Line-of-Sight (CFLOS) probability. In the system under study it is further assumed that the attenuation due to ice clouds is compensated by a static margin. For modeling the CFLOS on multiple spatially separated links, the methodology presented in [21] and [27] is used. In this latter model, time series of ILWC are generated for the region of the UT using multi-dimensional SDEs in order to also capture the spatial correlation of ILWC. After generating time series of liquid water content over threedimensions and taking into account the elevation angle of the link, the probability (or time percentage) that the slant path propagates through clouds can be obtained.

\section{Physical Layer Performance}

In this section, we address the physical layer optimization to develop the necessary inputs for the system capacity simulation. In terms of physical layer techniques, we employ advanced distortion mitigation techniques at the transmitter and at the receiver. Despite their sophistication, the employed techniques are within the reach of current processing technology. To reflect the system scenario that is being considered for the Q-band system, the overall available transponder bandwidth of $833 \mathrm{MHz}$ is split in two carriers for technology reasons (otherwise the sampling rate is considered overwhelming). Hence, we consider a scenario with two carriers per transponder (beam), and, in accordance with DVB-S2(X), we adopt phase shift keying (PSK) and amplitude and phase shift keying (APSK) constellations. At the transmitter side (GW), to mitigate the amount of non-linear High Power Amplifier (HPA) distortions and intermodulation due to the presence of the two carriers, we adopt the advanced iterative multicarrier predistortion scheme described in [28]. At the receiver a sufficient statistic for detection is extracted from the received signal by using oversampling at the output of a proper frontend filter [29]. For each carrier, a fractionally-spaced minimum mean-square error equalizer acts as an adaptive filter, allowing the receiver to operate in the absence of any knowledge about the channel model [30]. At this point, two alternative detection schemes can be envisaged:

1) Each of the two carriers is detected by means of a single-user detector (SUD). This represents the simplest solution from the point of view of the receiver complexity. We adopt a symbol-by-symbol SUD, which does not take into account the channel memory; this is because, in a multicarrier scenario, the main impairment is the interference from the adjacent channel, and not the memory effects introduced by the channel. This 
choice will allow us to evaluate the performance of the detection scheme also for high-order constellations.

2) Both carriers are jointly detected with a multiuser detector (MUD). This solution is expected to better cope with the interchannel interference, at the price of an increased complexity of the receiver [31]. To further increase the performance, we will use a MUD that takes into account one sample of the channel memory. To determine the target channel response for the detector, we will resort to an adaptive channel shortening filter [32], which processes the output of the equalizers and computes the optimal channel response for a given length (one in this case) of the interference to be considered by the detector. The adopted transponder model is that foreseen by the DVBS2 standard [5], composed of an input multiplexer (IMUX) filter, which has the purpose of removing adjacent channels, HPA and an output multiplexer (OMUX) filter, which reduces the spectral broadening caused by the nonlinear amplifier. The signal is further corrupted in the downlink by an additive white Gaussian noise process with one-sided power spectral density $N_{0}$.

In the described scenario, we will evaluate the spectral efficiency (SE) achieved by the practical low-density parity-check (LDPC) codes foreseen by the DVB-S2(X) standards [5], [6]. The SE is defined as

$$
\mathrm{SE}=\frac{r \log _{2} M}{T B} \quad[\mathrm{bit} / \mathrm{s} / \mathrm{Hz}]
$$

where $r$ is the rate of the adopted binary code, $M$ is the cardinality of the adopted constellation, $T$ is the symbol interval, and $B=833 \mathrm{MHz}$ is the $-3 \mathrm{~dB}$ bandwidth of the OMUX filter. The SE is computed with reference to a target packet error rate of $10^{-3}$ and it is represented in the Shannon plane as a function of $P_{\text {sat }} / N$, where $P_{\text {sat }}$ is the saturation power of the HPA, and $N=N_{0} B$ is the noise power in the considered bandwidth. An alternative figure of merit, useful to evaluate the performance of practical codes, is the achievable spectral efficiency (ASE), which represents the maximum SE that can be achieved by any practical MODCOD employing joint detection and decoding. It can thus be computed with no reference to a practical coding scheme. The ASE is defined as

$$
\mathrm{ASE}=\frac{I_{R}}{T B} \quad[\mathrm{bit} / \mathrm{s} / \mathrm{Hz}]
$$

where $I_{R}$ is the achievable information rate, which, for a system with memory, can be computed by means of the Monte Carlo method described in [33].

The aim of this section is to optimize the transmission parameters (i.e., the symbol rate, the frequency spacing between the two carriers, and the back-off of the HPA) of the described system to maximize the ASE with the two receiver structures under consideration. The MODCODs that result from this optimization are then used as input into the system level simulations. Fig. 4 reports the ASE for modulation formats with cardinality ranging from 2 to 64, with and without the use of the predistortion algorithm (pred. and no pred. curves, respectively), using the SUD receiver architecture. We point out that these curves are fully optimized, meaning that, for each value of $P_{\text {sat }} / N$, a different value of symbol rate and

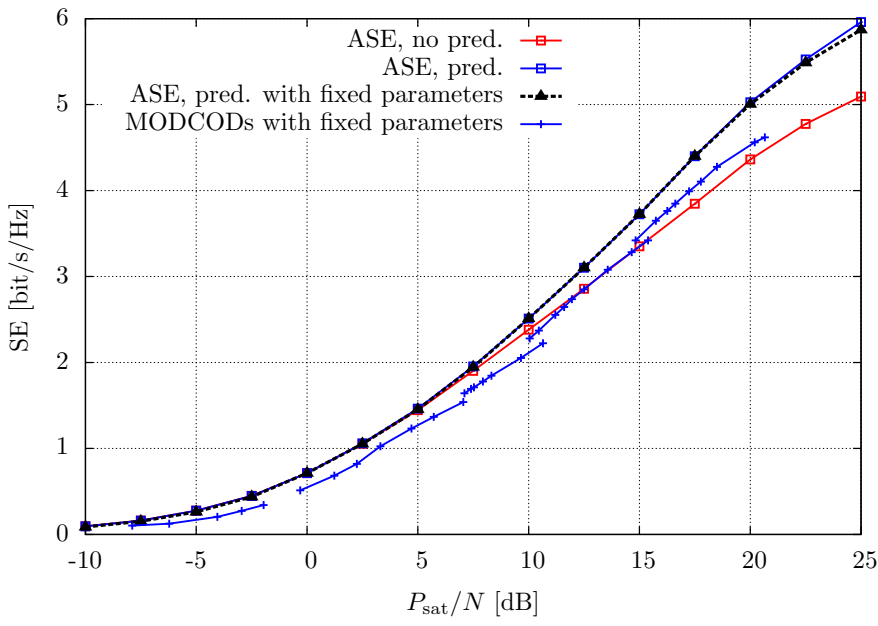

Fig. 4: Performance comparison with and without the predistorter and MODCODs spectral efficiency, using the SUD receiver structure.

TABLE II: Details of the selected MODCODs

\begin{tabular}{|c|c|c|c|c|c|c|}
\hline$\#$ & Mod. & Rate & $\begin{array}{c}E_{\mathrm{s}} / N_{0} \\
{[\mathrm{~dB}]}\end{array}$ & $\begin{array}{c}P_{\text {sat }} / N \\
{[\mathrm{~dB}]}\end{array}$ & $\begin{array}{c}\text { SE } \\
{[\mathrm{bit} / \mathrm{s} / \mathrm{Hz}]}\end{array}$ & $\begin{array}{c}\text { OBO } \\
{[\mathrm{dB}]}\end{array}$ \\
\hline \hline 15 & 8 PSK & $100 / 180$ & 6.36 & 7.53 & 1.7105 & 1.06 \\
\hline 16 & 8 PSK & $104 / 180$ & 6.77 & 7.94 & 1.7789 & 1.06 \\
\hline 17 & $8 \mathrm{PSK}$ & $3 / 5$ & 7.13 & 8.32 & 1.8474 & 1.08 \\
\hline 18 & $8 \mathrm{PSK}$ & $2 / 3$ & 7.97 & 9.65 & 2.0526 & 1.57 \\
\hline 19 & 8 PSK & $13 / 18$ & 8.97 & 10.65 & 2.2237 & 1.57 \\
\hline 20 & 16 APSK & $100 / 180$ & 8.22 & 10.05 & 2.2807 & 1.72 \\
\hline 21 & 16 APSK & $104 / 180$ & 8.63 & 10.46 & 2.3719 & 1.72 \\
\hline 22 & 16 APSK & $28 / 45$ & 9.37 & 11.2 & 2.5544 & 1.72 \\
\hline 23 & 16 APSK & $116 / 180$ & 9.77 & 11.6 & 2.6456 & 1.72 \\
\hline 24 & 16 APSK & $2 / 3$ & 10.12 & 11.95 & 2.7368 & 1.72 \\
\hline 25 & 16 APSK & $25 / 36$ & 10.59 & 12.5 & 2.8509 & 1.8 \\
\hline 26 & 16 APSK & $3 / 4$ & 11.66 & 13.57 & 3.0789 & 1.8 \\
\hline
\end{tabular}

frequency spacing is selected. However, in a scenario in which we are constrained to adopt a unique symbol rate, we can resort to the practical solution of fixing a single parameters configuration. This solution is not optimal from the theoretical point of view, but we see from the black curve in Fig. 4 that the losses with respect to the fully optimized curve are very limited. This configuration corresponds to a symbol rate of $427.46 \mathrm{Mbaud}$ and frequency spacing $449.38 \mathrm{MHz}$, i.e., the values optimized for an intermediate value of $P_{\text {sat }} / N$. Moreover, we have computed the SE achieved by several practical MODCODs with these parameters, also reported in Fig. 4. We see that the almost all MODCODs are in the range $1-1.5 \mathrm{~dB}$ from the theoretical ASE. The details of a subset of the selected MODCODs are reported in Table II, where $E_{\mathrm{s}}$ is the average energy per symbol, and OBO is the output back-off of the HPA. The number in the first column will be used to identify the MODCODs in the next sections.

Finally, we evaluate the performance of the MUD in the considered scenario. For complexity reasons, we limit the analysis to constellations up to 16APSK. Fig. 5 compares the ASE of the MUD with that of the SUD, with and without the use of the predistortion algorithm. The optimized parameters for the MUD are reported in Table III. We see that the SUD and the MUD have a similar performance in the medium SNR range, while the MUD allows to adopt significantly higher symbol rate values at high SNR. Higher ASE values 


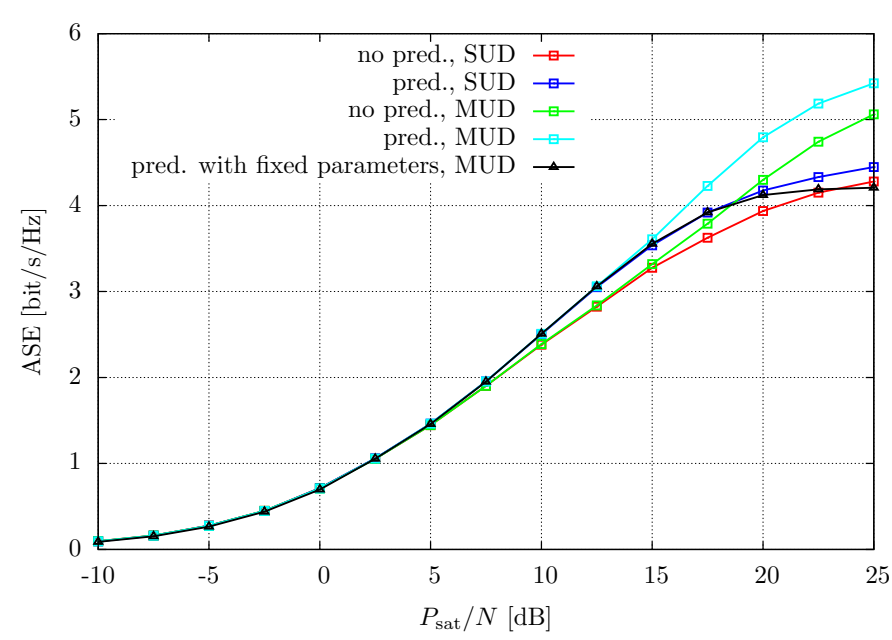

Fig. 5: ASE comparison with and without the predistorter and using the MUD receiver structure.

TABLE III: Optimized parameters for system adopting the predistorter and the MUD

\begin{tabular}{|c|c|c|c|c|c|c|}
\hline Mod. & $\begin{array}{c}E_{\mathrm{S}} / N_{0} \\
{[\mathrm{~dB}]}\end{array}$ & $\begin{array}{c}P_{\text {sat }} / N \\
{[\mathrm{~dB}]}\end{array}$ & $\begin{array}{c}\text { ASE } \\
{[\mathrm{bit} / \mathrm{s} / \mathrm{Hz}]}\end{array}$ & $\begin{array}{c}\text { Symb. rate } \\
{[\mathrm{Mbaud}]}\end{array}$ & $\begin{array}{c}\text { Freq. sp. } \\
{[\mathrm{MHz}]}\end{array}$ & $\begin{array}{c}\text { OBO } \\
{[\mathrm{dB}]}\end{array}$ \\
\hline \hline 8PSK & -10.94 & -10.0 & 0.0946 & 416.50 & 405.54 & 0.94 \\
\hline 8PSK & -5.82 & -5.0 & 0.2743 & 405.54 & 405.54 & 0.94 \\
\hline 8PSK & -1.05 & 0.0 & 0.7091 & 427.46 & 427.46 & 0.94 \\
\hline 8PSK & 3.87 & 5.0 & 1.4636 & 427.46 & 427.46 & 1.02 \\
\hline 16APSK & 8.09 & 10.0 & 2.5079 & 438.42 & 449.38 & 1.69 \\
\hline 16APSK & 11.55 & 15.0 & 3.6076 & 548.03 & 591.87 & 2.25 \\
\hline 16APSK & 15.29 & 20.0 & 4.7940 & 580.91 & 580.91 & 3.26 \\
\hline 16APSK & 18.69 & 25.0 & 5.4216 & 591.87 & 591.87 & 4.79 \\
\hline
\end{tabular}

could be achieved with the MUD by transmitting completely overlapped signals with bandwidth comparable with that of the OMUX filter. However, this would lead to an excessively high sampling rate, which is not feasible for the current technology. Moreover, we have to mention that standard DVBS2(X) LDPC codes are not designed to work together with a MUD, and they would lead to poor performance in this scenario. To fully exploit the characteristics of the MUD, a new code design is required [34]. The selected parameters are symbol rate equal to 438.42 Mbaud and frequency spacing equal to $449.38 \mathrm{MHz}$.

\section{System Simulation Results and Discussion}

In this section, the simulation methodology and step-by-step algorithms are given for the calculation of capacity time series and then of the required capacity statistics based on the tools developed in the previous sections. The results are presented in terms of histogram of the selected MODCODs and outage capacity statistics for four of the eight ground stations in Table I.

\section{A. Simulation Methodology}

Starting with the capacity time series of the orbital diversity system operating in Q-band, the following step-by-step algorithm is used:

- At first, the time series of sub-satellite points are generated for each of the eight satellites. Toward this, the Systems Tool Kit of AGI is used [35].
- From the subsatellite points, the elevation angle from every ground station to every satellite is calculated

- For every simulation sample, the separation angle between the satellites is calculated. Then, using also as input the elevation angle time series, the correlation coefficient for every attenuation factor is calculated.

- Then, the free space losses for every sample are calculated and the channel time series are generated

- In the visibility area of the ground station the link with the least total attenuation, i.e. free space losses plus atmospheric attenuation (which follows the methodology of Section III), is selected

- For the selected link, the SNR is then calculated.

- From the SNR time series, using the MODCOD thresholds of Section IV, the capacity time series are calculated

Moreover, it must be noted that in this work an ideal orbital diversity scheme is actually simulated considering that every time instance the ground stations can receive the same signal from both satellites assuming no delay for switching and always spare capacity to be used by the alternative satellite. In a non-ideal scenario, an algorithm to trigger the orbital diversity for a user needs to be developed and also to take into account any delays resulting from the switching and the extra signal propagation, either through Inter-Satellite Links or from the MEO GW to the second satellite. However, such analysis is out of scope of the paper considering the state of the art approaches of MEO satellite systems. For the site diversity scenario, we have assumed that the two ground stations are at the same altitude although they are separated by $20 \mathrm{~km}$. For the generation of capacity time series, similarly to the orbital diversity after the calculation of elevation angle time series, the following step-by-step algorithm is used:

- Using the separation distance between the ground stations, spatial correlation coefficients for all the attenuation factors are calculated

- Using the elevation angle time series and the spatial correlation coefficients, the channel time series are generated as explained in Section III.

- The link with the minimum atmospheric attenuation is selected for the communication between Earth and space

- For the selected link, the SNR is then calculated, using the link budget parameters and the free space losses

- From the SNR time series, using the MODCOD thresholds, the capacity time series are calculated

As an illustration, in Fig. 6, the time series of elevation angle for the UT in Hawaii are shown for the whole MEO constellation, when orbital diversity is used and when it is not used. From the figure it is evident that the link with the highest elevation angle (single link case) is not always selected as it does not give always the lowest attenuation.

In Fig. 7, the total attenuation, i.e. total atmospheric attenuation plus free space losses, time series are shown for the same time period as in Fig. 6 for all considered links. In particular, for every sample the total attenuation in the link between the UT and the visible satellites is shown. The same colour code of Fig. 6 is also kept in Fig. 7. We repeat the same type of plots for the site diversity system in Figs. 8 and 9. In Fig. 8, 


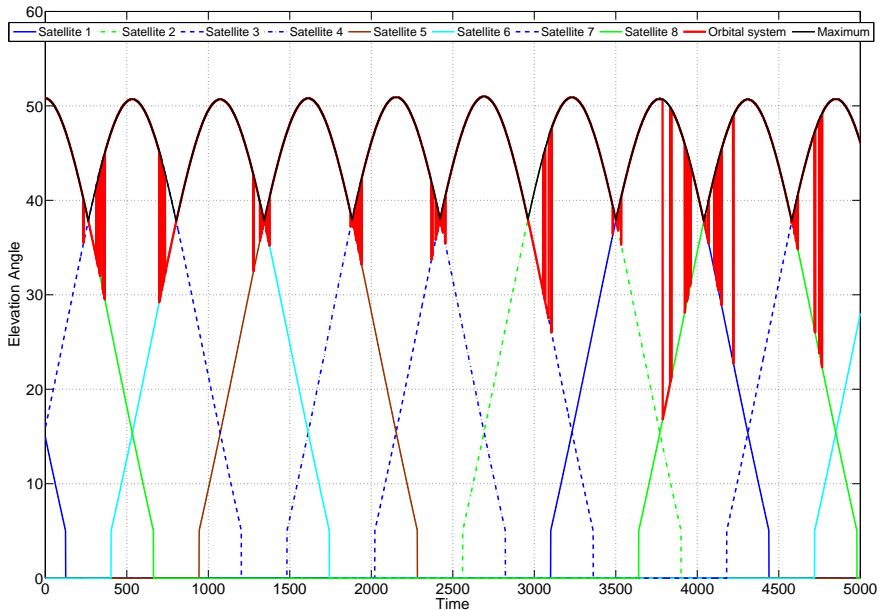

Fig. 6: Time Series of elevation angle for the orbital diversity system

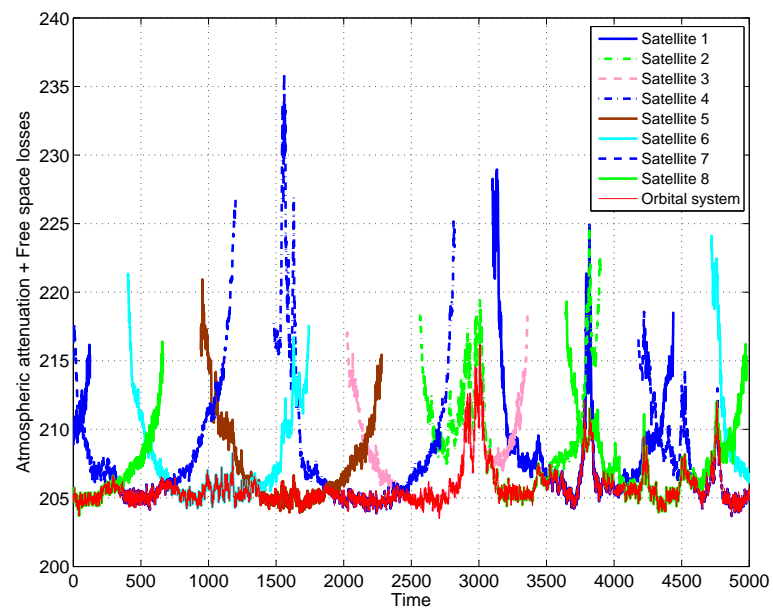

Fig. 7: Time Series of atmospheric losses and free space losses for the orbital system

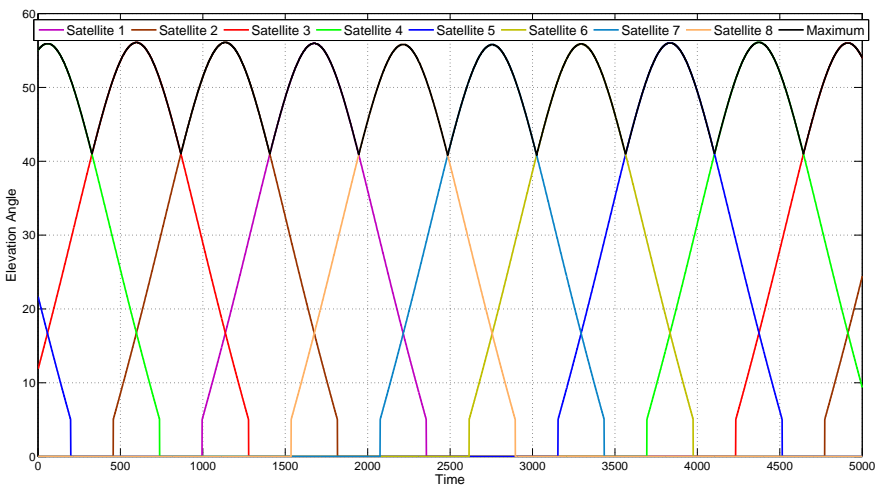

Fig. 8: Time Series of elevation angle for the site diversity system

the black line gives the elevation angle of the system, which is the maximum elevation angle for the satellites in the visibility area of the UTs. In Fig. 9, the atmospheric attenuation time series are shown for the site diversity system, with the black line representing the attenuation value the system is suffering from.

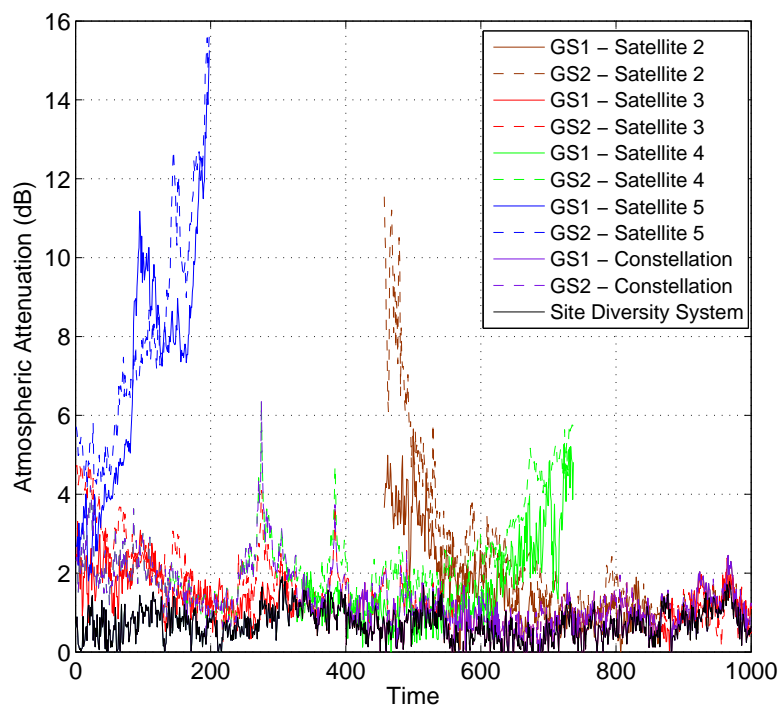

Fig. 9: Time Series of atmospheric losses for the site diversity system

\section{B. Optical Links}

The requirement that all systems under investigation must comply with is the target of $99.9 \%$ availability. This is quite a challenge for optical communications due to the deteriorating effect of clouds and spatial diversity between multiple optical ground stations at large distances is absolutely needed. For the stations given in Table I, we will consider the use of spatial diversity reception in order to achieve $99.9 \%$ availability for each location. Due to the significant separation distances required, the geometry of the site diversity network needs to obey specific geographical restrictions: as an example for Nemea and Sintra an effort was made to keep the stations within the countries borders. For Hortolandia, spreading the stations in different countries could not be avoided. Lima on the other hand, experiences low cloud probability due to the its high altitude.

In Table IV, the number of stations needed for every location in order to meet the target availability, i.e. CFLOS, higher than $99.9 \%$ is given. Here, it must be noted that the separation distance between the stations is higher than 100 $\mathrm{km}$, a fact that reveals some of the operational difficulties of setting up a high availability optical ground network. As an example, Fig. 10 shows the Earth stations for the site diversity system deployed in Pakistan, being the main station located in Karachi, Pakistan. For the scheme, the distance between Karachi and Stations 2, 3, 4 and 5 is approximately $303 \mathrm{~km}$, $440 \mathrm{~km}, 587 \mathrm{~km}$ and $447 \mathrm{~km}$, respectively.

\section{Capacity Statistics}

In this section, we report the numerical results from processing the capacity time series of the next generation MEO trunking system operating at Q-band; for the three different diversity techniques, i.e., the single link (no diversity) scenario, the orbital diversity scenario and the site diversity scenario. The single link scenario at Q-band has been studied in [9]. 
TABLE IV: Number of ground stations considered for each region and the derived availability

\begin{tabular}{|c|c|c|}
\hline Region & Number of stations & Availability [\%] \\
\hline \hline Nemea & 8 & 99.906 \\
\hline Karachi & 5 & 99.94 \\
\hline Vernon & 8 & 99.94 \\
\hline Dubbo & 7 & 99.929 \\
\hline Sintra & 8 & 99.92 \\
\hline Hortolandia & 9 & 99.93 \\
\hline Lima & 4 & 99.93 \\
\hline
\end{tabular}

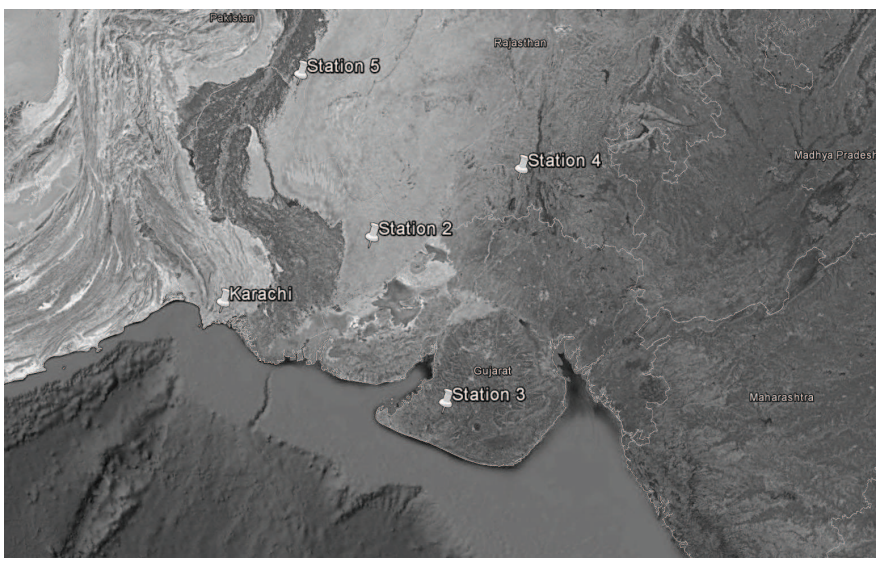

Fig. 10: Geometry of diversity scenario including Karachi

However, here also the results for more stations are presented. All the system capacity results presented hereinafter assume $\mathrm{ACM}$ and refer to the cumulative capacity from 10 user beams per MEO satellite. Results are presented with respect to the two UT antenna diameters that have been considered, i.e., 1.8 $\mathrm{m}$ and $3.5 \mathrm{~m}$, and compared with a Ka-band constellation operating with the same parameters (see [8]).

The capacity results are reported for an interesting subset of those locations listed in Table I, in terms of probability that capacity falls below a certain capacity threshold, expressed in bits per second (bps) and in terms of probability of selecting one of the MODCOD schemes for the complete set defined in Section IV.

In Fig. 11 the Cumulative Distribution Function (CDF) of the achieved capacity is reported for the 4 selected locations (Lima, Vernon, Sintra, Hortolandia), while in Fig. 12, the probability of selecting each MODCOD schemes is reported. It is worth noticing that while the outage capacity figures show a comparison between the Q-band and the Ka-band systems, in case of MODCOD scheme probability of selection we report only the Q-band results; this is because the used MODCOD schemes in the two bands are different [8]. The MODCOD index refers to the index in the MODCOD set listed in Table II.

By analyzing the numerical results, it is observed that there are some dominant MODCOD schemes that are selected with a high probability during a pass of the MEO satellite over the UT; their value depends on the location and on the antenna size but the amount of used MODCODs is quite similar in all the considered cases. There is a maximum possible MODCOD and the selection of the lowest schemes is taking place during the very short deep attenuation intervals that

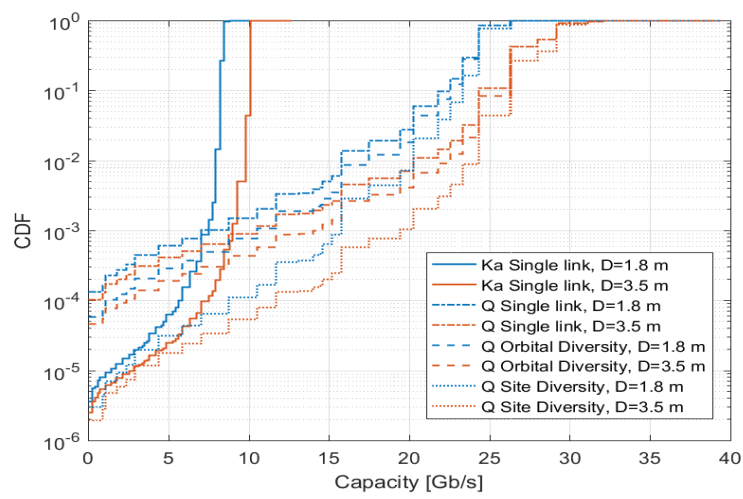

(a) Lima,Peru

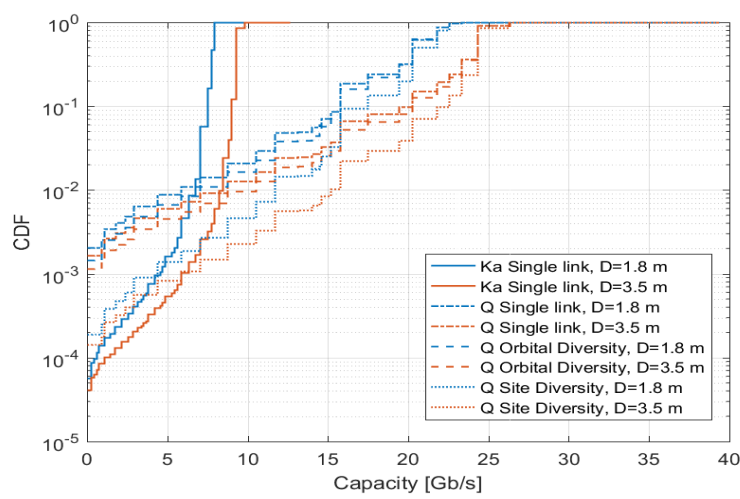

(b) Vernon, TX, USA

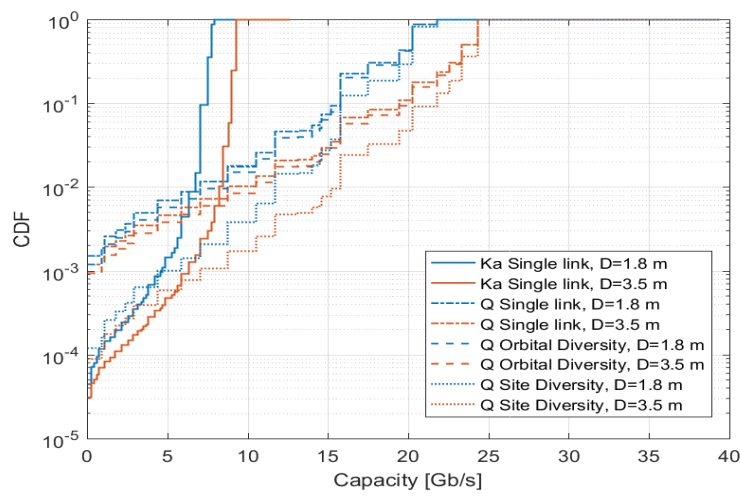

(c) Sintra,Portugal

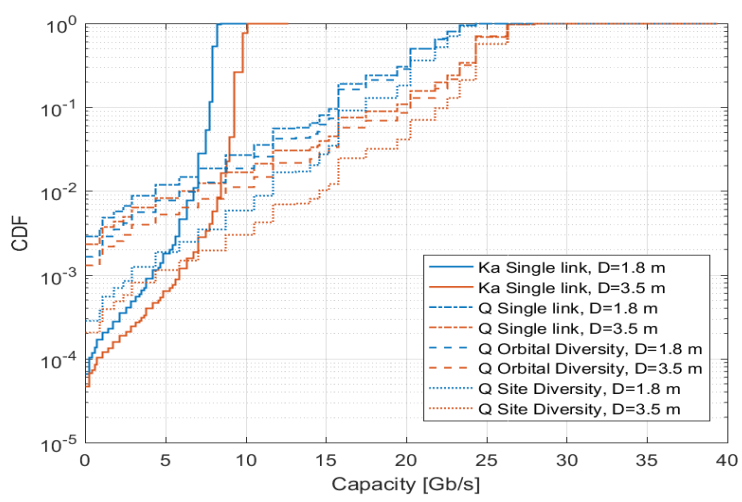

(d) Hortolandia,Brazil

Fig. 11: CDF of the capacity in the considered bands and diversity schemes for the considered locations. 


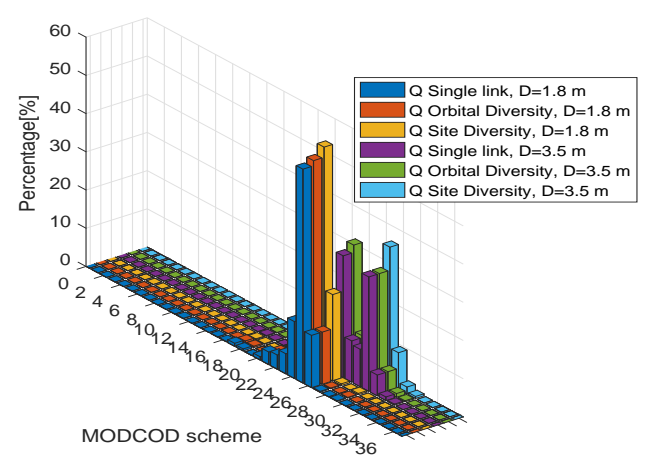

(a) Lima,Peru

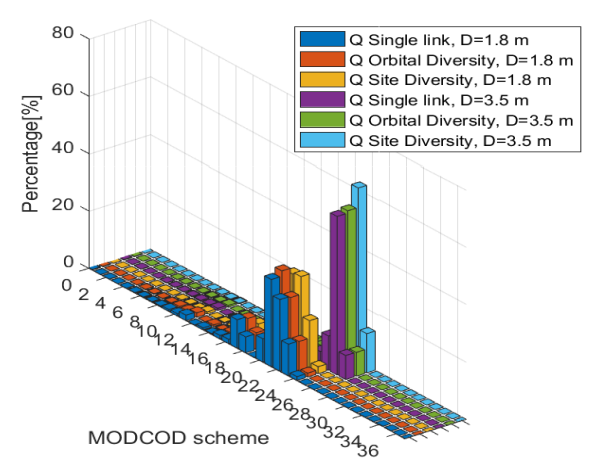

(b) Vernon, TX, USA

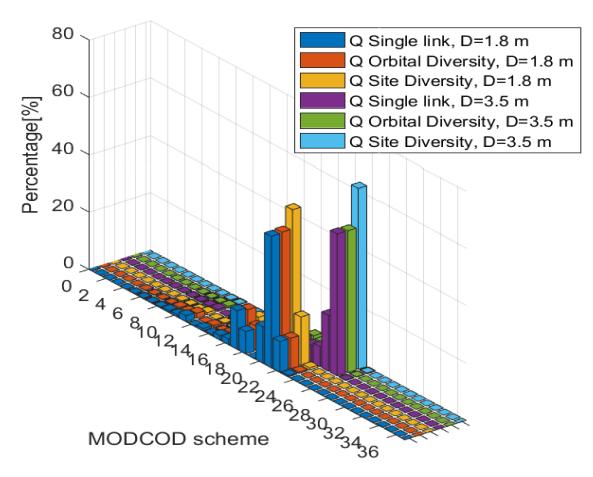

(c) Sintra,Portugal

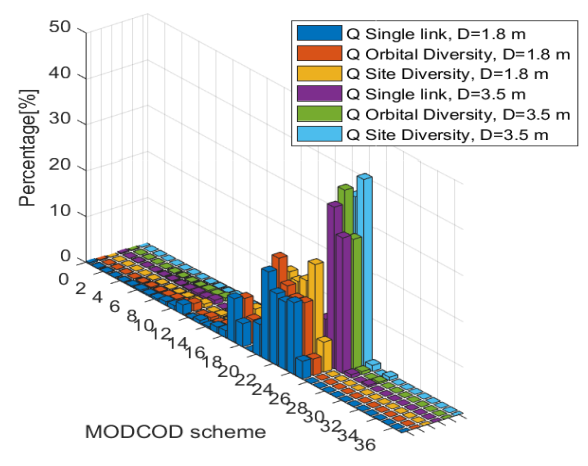

(d) Hortolandia,Brazil

Fig. 12: Histograms of the selected MODCOD schemes in the Q band in the different diversity schemes for the considered locations. occur for a small percentage of time. This is also highlighted in the CDF of the capacity where the logarithmic scale of the CDF allows noticing that very low probability values are possible, especially for lower MODCODs.

The CDFs of Fig. 11 allow understanding the capacity offered per MEO satellite belonging to a multi-satellite constellation by comparing the performance in $\mathrm{Ka}$ and $\mathrm{Q}$ bands. In case of $\mathrm{Q}$ band it is possible to achieve an impressive capacity in case of clear sky conditions, which corresponds to a level of about $95 \%$. In this case the Q-band system with single link achieves between $12 \mathrm{~Gb} / \mathrm{s}$ in Hortolandia to $20 \mathrm{~Gb} / \mathrm{s}$ in Lima for $1.8 \mathrm{~m}$ receiver antennas, while, in case of $3.5 \mathrm{~m}$ antenna, it is possible to achieve between $16 \mathrm{~Gb} / \mathrm{s}$ in Vernon, Sintra and Hortolandia to $24 \mathrm{~Gb} / \mathrm{s}$ in Lima. Such capacity values are about double with respect to the capacity values obtained in the $\mathrm{Ka}$ band for the same locations and antenna sizes.

On the other side it has to be noticed that the outage probability is higher as well as the capacity for the full availability (i.e., 99.9\%) is lower with respect to the Kaband; this is due to the higher influence of the atmospheric attenuation phenomena at higher frequencies. Nevertheless, the lower capacity offered at high availability is not important due to the very limited periods that these intense phenomena occurs. What is more critical to note is that availability down to $99.9 \%$ can be preserved in Q-band sometimes even with a single station. By exploiting multiple sites at the ground, i.e., implementing a site diversity, it is indeed possible to reduce remarkably the outage probability.

Finally, for the optical system capacity calculation, it should be pointed out that one redundant optical terminal on board is needed for each active one. This happens because for the site diversity scheme to achieve transparent handover without breaking the link, the alternative optical terminal needs to already have set up (point, acquire and track) the link to a cloud free station. Therefore, since the number of terminals on the satellite is 8 , we can only assume that 4 of them are active. Based on our earlier assumptions, this means that the total throughput of the optical system is 4 times 20 , equal to 80Gbps per satellite.

\section{CONCLUSION}

In this study various advanced options for a next generation MEO system were evaluated considering a constellation of 8 satellites and eight possible UT locations at different climatic areas. The outage capacity offered by 5 different system scenarios was evaluated keeping the satellite power consumption fixed to $1300 \mathrm{~W}$, the mass similar, the latency less than 150 ms and the availability better than $99.9 \%$. The RF UTs were considered with two kinds of antenna classes, namely $1.8 \mathrm{~m}$ and $3.5 \mathrm{~m}$. Four RF scenarios were compared one at Ka-band (reference) and three at $\mathrm{Q}$ band (advanced system) without diversity, one using orbital diversity and one for site diversity. The fifth scenario considered wavelengths in the optical range necessarily applying site diversity.

From the capacity results it was found that on average there is a $100 \%$ increase in system throughput at $\mathrm{Q}$ band in comparison to a clear sky reference scenario in Ka-band. 
Moreover, in Q band the mean system throughput at clear sky is close to 15 Gbps per satellite for the ten user beams. However, in the single link case the $99.9 \%$ availability is not always assured. Using orbital diversity again the $99.9 \%$ availability is not guaranteed for all the stations but there is an increase in both the availability and capacity. Finally, with site diversity the availability is always on target. Here it must be noted that orbital diversity can be already implemented with the existing O3b system since every UT has already two antennas. For the optical system in most locations more than 6 ground stations are required to achieve the target availability of $99.9 \%$ and only when these are available close to $80 \mathrm{Gbps}$ can be delivered.

The study of the use of dual polarization on each beam is an interesting future expansion of the current work, since the use of this technique along with Multiple Input Multiple Output (MIMO) techniques could increase the capacity used in the RF MEO SatCom systems [36]. Moreover, algorithms to trigger the satellite diversity incorporating the delays due to the switch of the user from one satellite to another and their impact on the capacity statistics must be studied.

\section{ACKNOWLEDGMENT}

This research has been financed by the European Space Agency under the framework of SatNEx IV project.

\section{REFERENCES}

[1] O3b satellite networks. Accessed May 2014. [Online]. Available: http://www.o3bnetworks.com/o3b-advantage/our-technology

[2] S. H. Blumenthal, "Medium Earth orbit Ka-band satellite communication systems," in MILCOM 2013 - 2013 IEEE Military Communications Conference, Nov. 2013, pp. 273-277.

[3] Laser Light Communications. Accessed Feb 2016. [Online]. Available: http://www.laserlightcomms.com/index.php

[4] A. Panagopoulos, P.-D. M. Arapoglou, and P. Cottis, "Satellite communications at $\mathrm{KU}, \mathrm{KA}$, and $\mathrm{V}$ bands: Propagation impairments and mitigation techniques," Communications Surveys Tutorials, IEEE, vol. 6, no. 3, pp. 2-14, Third 2004.

[5] "Second generation framing structure, channel coding and modulation systems for broadcasting, interactive services, news gathering and other broadband satellite applications, part I: DVB-S2," ETSI EN 302 307-1 Digital Video Broadcasting (DVB).

[6] "Second generation framing structure, channel coding and modulation systems for broadcasting, interactive services, news gathering and other broadband satellite applications, part II: S2-extensions (DVB-S2X)," ETSI EN 302 307-2 Digital Video Broadcasting (DVB).

[7] H. Fenech, S. Amos, A. Tomatis, and V. Soumpholphakdy, "High throughput satellite systems: An analytical approach," IEEE Transactions on Aerospace and Electronic Systems, vol. 51, no. 1, pp. 192-202, January 2015.

[8] C. Kourogiorgas, D. Tarchi, A. Ugolini, P.-D. Arapoglou, A. D. Panagopoulos, G. Colavolpe, and A. Vanelli-Corrali, "System capacity evaluation of DVB-S2X based medium earth orbit satellite network operating at ka-band," in in ASMS/SPSC Conference, Sep. 2016.

[9] _ - "Performance evaluation of DVB-S2X based MEO satellite networks operating at Q-band," in IEEE GlobeCom 2016, Dec. 2016.

[10] SatNEx IV. Accessed Sep 2016. [Online]. Available: http://www.satnex4.org/2-uncategorised/11-welcome

[11] "Radio regulations - articles," ITU-R., 2012.

[12] R. Lange, F. Heine, M. Motzigemba, M. Lutzer, and R. Meyer, "Roadmap to wide band optical geo relay networks," in MILCOM 2012 2012 IEEE Military Communications Conference, Oct. 29 - Nov.1 2012.

[13] "Tropospheric attenuation time series synthesis," ITU-R. P. 1853-1, 2012.

[14] C. Kourogiorgas and A. Panagopoulos, "A rain attenuation stochastic dynamic model for LEO satellite systems above $10 \mathrm{GHz}$," Vehicular Technology, IEEE Transactions on, vol. 64, no. 2, pp. 829-834, Feb. 2015.
[15] I. Karatzas and S. E. Shreve, Brownian Motion and Stochastic Calculus. Springer-Verlag, 2005.

[16] G. A. Karagiannis, A. D. Panagopoulos, and J. D. Kanellopoulos, "Multidimensional rain attenuation stochastic dynamic modeling application to Earth space diversity systems," Antennas and Propagation, IEEE Transactions on, vol. 60, no. 11, pp. 5400-5411, Nov. 2012.

[17] "Propagation data and prediction methods required for the design of Earth-space telecommunication systems," ITU-R. P. 618-12, 2015.

[18] A. D. Panagopoulos and J. D. Kanellopoulos, "Prediction of triple-orbital diversity performance in earth-space communication," International Journal of Satellite Communications, vol. 20, no. 3, pp. 187-200, 2002. [Online]. Available: http://dx.doi.org/10.1002/sat.720

[19] "Attenuation due to clouds and fog," ITU-R. P. 840, 2013.

[20] L. Luini and C. Capsoni, "Modeling high-resolution 3-D cloud fields for earth-space communication systems," Antennas and Propagation, IEEE Transactions on, vol. 62, no. 10, pp. 5190-5199, Oct. 2014.

[21] N. K. Lyras, C. I. Kourogiorgas, and A. D. Panagopoulos, "Cloud attenuation statistics prediction from ka-band to optical frequencies: Integrated liquid water content field synthesizer," IEEE Transactions on Antennas and Propagation, vol. 65, no. 1, pp. 319-328, Jan 2017.

[22] N. Jeannin et al., "A space time channel model for the simulation of total attenuation fields," in ESA Workshop on Radiowave Propagation, Nov. 2011.

[23] "Attenuation by atmospheric gases," ITU-R. P. 676, 2012.

[24] N. Jeannin, L. Feral, H. Sauvageot, and L. Castanet, "Statistical distribution of integrated liquid water and water vapor content from meteorological reanalysis," Antennas and Propagation, IEEE Transactions on, vol. 56, no. 10, pp. 3350-3355, Oct. 2008.

[25] C. Kourogiorgas and A. D. Panagopoulos, "A tropospheric scintillation time series synthesizer based on stochastic differential equations," in 2013 Joint Conference: 19th Ka and Broadband Communications, Navigation and Earth Observation Conference and 31st AIAA ICSSC, Oct. 2013.

[26] E. Matricciani, M. Mauri, and C. Riva, "Scintillation and simultaneous rain attenuation at $49.5 \mathrm{ghz}$," in Antennas and Propagation, 1995., Ninth International Conference on (Conf. Publ. No. 407), vol. 2, Apr 1995, pp. $165-168$ vol.2.

[27] N. Lyras, C. Kourogiorgas, and A. D. Panagopoulos, "Joint statistics of cloud attenuation induced on multiple optical satellite links," in 21th Ka band and Broadband Communications, Navigation and Earth Observation Conference, Oct. 2015.

[28] B. Beidas, R. Seshadri, and N. Becker, "Multicarrier successive predistortion for nonlinear satellite systems," IEEE Trans. on Commun., vol. 63, no. 4, pp. 1373-1382, Apr. 2015.

[29] H. Meyr, M. Oerder, and A. Polydoros, "On sampling rate, analog prefiltering, and sufficient statistics for digital receivers," IEEE Trans. on Commun., vol. 42, pp. 3208-3214, Dec. 1994.

[30] A. Ugolini, A. Modenini, G. Colavolpe, V. Mignone, and A. Morello, "Advanced techniques for spectrally efficient DVB-S2X systems," Intern. J. of Satellite Communications and Networking, vol. 34, pp. 609623, Sept./Oct. 2016, article first published online: Sept. 2015.

[31] S. Verdú, Multiuser Detection. Cambridge, UK: Cambridge University Press, 1998

[32] A. Modenini, F. Rusek, and G. Colavolpe, "Adaptive rate-maximizing channel-shortening for ISI channels," IEEE Commun. Letters, vol. 19, no. 12, pp. 2090-2093, Dec. 2015.

[33] D. M. Arnold, H.-A. Loeliger, P. O. Vontobel, A. Kavčić, and W. Zeng, "Simulation-based computation of information rates for channels with memory," IEEE Trans. Inform. Theory, vol. 52, no. 8, pp. 3498-3508, Aug. 2006.

[34] S. ten Brink, G. Kramer, and A. Ashikhmin, "Design of low-density parity-check codes for modulation and detection," IEEE Trans. Commun., vol. 52, pp. 670-678, Apr. 2004.

[35] "AGI STK." [Online]. Available: https://www.agi.com/products/byproduct-type/applications/stk/

[36] P. D. Arapoglou, K. Liolis, M. Bertinelli, A. Panagopoulos, P. Cottis, and R. D. Gaudenzi, "MIMO over satellite: A review," IEEE Cоттиnications Surveys Tutorials, vol. 13, no. 1, pp. 27-51, First 2011. 


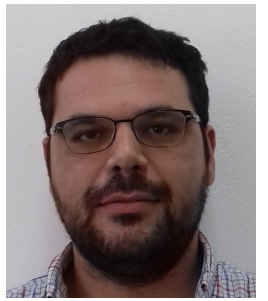

Charilaos I. Kourogiorgas was born in Athens, Greece, on July 6, 1985. He received the Diploma Engineering in electrical and computer engineering from the National Technical University of Athens (NTUA), Athens, Greece, in 2009. From October 2009 to June 2011 he was with Department of Electromagnetism and Radar at ONERA, Toulouse, France. In May 2015, he obtained the PhD in electrical and computer engineering from the National Technical University of Athens. From January to October 2016 he served in the CyberSecurity unit of the Hellenic Army IT Support Center. He has published more than 50 papers in international refereed journals and conferences. He has been awarded with the Chorafas Scholarship for his PhD studies and he is the recipient of URSI Young Scientist Award for 2014. He is a member of Technical Chamber of Greece. His research interests include channel modeling for satellite and terrestrial communication systems and the evaluation of system's performance.

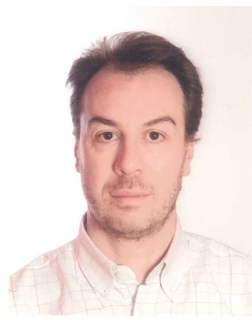

Daniele Tarchi (S'99-M'05-SM'12) is an assistant professor at the University of Bologna, Italy. He received M.Sc. in Telecommunications Engineering and $\mathrm{Ph} . \mathrm{D}$. in Computer and Telecommunications Engineering from the University of Florence, Italy, in 2000 and 2004, respectively. His research interests include the telecommunication area, with particular interests in resource allocation and link adaptation algorithms in wireless and satellite networks. He has been involved in several national projects as well as European projects, and has been active in several industry funded projects; he is now the local coordinator for the Italian National project Gaucho. He is author of more than 100 paper in international journals and conference proceedings. He is an Editorial Board member for IEEE Wireless Communications Letters, IEEE Transactions on Vehicular Technology, and IET Communications, and has been an Editorial Board member for Wiley Wireless Communication and Mobile Computing, Hindawi Journal of Engineering, and the Scientific World Journal, and has served as an Associate Editor for IEEE Transactions on Wireless Communications. $\mathrm{He}$ was Symposium Chair at IEEE Wireless Communications and Networking Conference 2011 and at IEEE GLOBECOM 2014, and Workshop Co-Chair at IEEE ICC 2015.

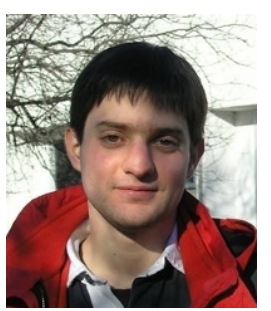

Alessandro Ugolini was born in Parma, Italy, in 1987. He received the Master's degree in Telecommunications Engineering (cum laude) from the University of Parma in 2012, and the Ph.D. degree in Information Technology from the same University in 2016. In 2012 he was awarded a National Inter-University Consortium for Telecommunications (CNIT) scholarship and a research grant funded by the Dipartimento di Ingegneria dell'Informazione (DII), University of Parma, for the study of synchronization algorithms for spectrally efficient systems. In 2016 he has been a postdoctoral researcher at the DII. Since 2017 he is a researcher at the Dipartimento di Ingegneria e Architettura of the University of Parma, in the SPADiC Lab research group. His main research interests include digital communications, applied information theory and synchronization. $\mathrm{He}$ has participated in several research projects funded by theEuropean Space Agency (ESA-ESTEC).

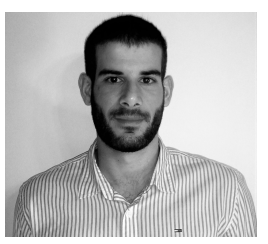

Nikolaos Lyras was born in Kalamata, Greece on June 2, 1991. He received the Diploma degree in electrical and computer engineering from the National Technical University of Athens (NTUA), Athens, Greece, in February 2015 where he is currently working toward the $\mathrm{Ph} . \mathrm{D}$. degree. He has been involved in ESA funded SATNEX IV and ONSET research projects while he has published more than 9 papers in international conferences and journals. His research interests include optical communication systems design, wireless and satellite communications networks and channel modeling for satellite and terrestrial communication systems.

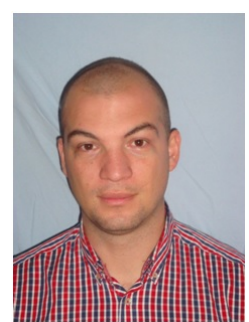

Pantelis-Daniel Arapoglou received the Diploma degree in electrical and computer engineering and the Dr.Eng. degree from the National Technical University of Athens (NTUA), Athens, Greece, in 2003 and 2007, respectively. From September 2008 to October 2010, he was involved in postdoctoral research on MIMO over satellite jointly supported by the NTUA and the European Space Agency Research and Technology Centre (ESA/ESTEC), The Netherlands. From October 2010 to September 2011, he was a Research Associate with the Interdisciplinary Centre for Security, Reliability and Trust (SnT), University of Luxembourg. Since September 2011, he has been a Communications System Engineer at ESA/ESTEC, where he is technically supporting R\&D activities and developments in the areas of satellite telecommunications, digital \& optical communications, and high data rate telemetry for Earth observation applications. Daniel was a recipient of the Ericsson Award of Excellence in Telecommunications for his diploma thesis in 2004 and of the URSI General Assembly Young Scientist Award in 2005. As a researcher, he has participated in the work of Study Group 3 of the ITU-R. Currently, he is following the CCSDS Optical Working Group.

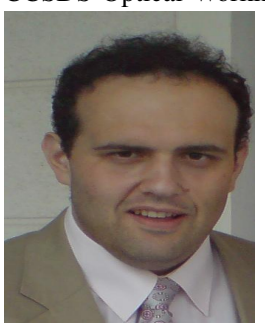

Athanasios D. Panagopoulos (S'98, M'02, SM'09): was born in Athens, Greece on January 26, 1975. He received the Diploma Degree in Electrical and Computer Engineering (summa cum laude) and the Dr. Engineering Degree from National Technical University of Athens (NTUA) in July 1997 and in April 2002. From May 2002 to July 2003, he served the Technical Corps of Hellenic Army. In September 2003, he joined School of Pedagogical and Technological Education, as part-time Assistant Professor. From January 2005 to May 2008, he was head of the Satellite Division of Hellenic Authority for the Information and Communication Security and Privacy. From May 2008 to May 2013 he was Lecturer in the School of Electrical and Computer Engineering of NTUA and now he is Assistant Professor. He has published more than 140 papers in International Journals and more than 190 papers in conference proceedings. $\mathrm{He}$ has also published more than 25 Book Chapters in International Books. He is the recipient of URSI General Assembly Young Scientist Award in 2002 and 2005. He is co-recipient of the Best Paper Awards in IEEE RAWCON 2006 and IEEE ISWCS 2015. His research interests include radio communication systems design, wireless and satellite communications networks and mobile computing technologies. He has participated to ITU-R and ETSI Study Groups, he is member of Technical Chamber of Greece and Senior Member of IEEE. He is Chairman of the IEEE Greek Communication Chapter. He serves on the editorial boards of International Journal of Antennas and Propagation, Elsevier Physical Communication. Finally, he is an Associate Editor of IEEE Transactions on Antennas and Propagation and an Associate Editor in IEEE Communication Letters.

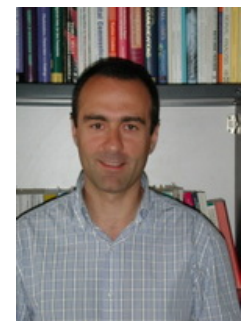

Giulio Colavolpe (S'96-M'00-SM'11) was born in Cosenza, Italy, in 1969. He received the Dr. Ing. degree in Telecommunications Engineering (cum laude) from the University of Pisa, Italy, in 1994 and the Ph.D. degree in Information Technologies from the University of Parma, Italy, in 1998. Since 1997, he has been at the University of Parma, Italy, where he is now Professor of Telecommunications at the Dipartimento di Ingegneria e Architettura (DIA). In 2000, he was Visiting Scientist at the Institut Eurécom, Valbonne, France. His research interests include the design of digital communication systems, adaptive signal processing (with particular emphasis on iterative detection techniques for channels with memory), channel coding and information theory. His research activity has led to more than 200 papers in refereed journals and in leading international conferences, and 18 industrial patents. He received the best paper award at the 13th International Conference on Software, Telecommunications and Computer Networks (SoftCOM'05), Split, Croatia, September 2005, the best paper award for Optical Networks and Systems at the IEEE International Conference on Communications (ICC 2008), Beijing, China, May 2008, and the best paper award at the 5th Advanced Satellite Mobile Systems Conference and 11th International Workshop on Signal Processing for Space Communications (ASMS\&SPSC 2010), Cagliari, Italy. He is currently serving as an Editor for IEEE Transactions on Communications. He also served as an Editor for IEEE Transactions on Wireless Communications and IEEE Wireless Communications Letters and as an Executive Editor for Transactions on Emerging Telecommunications Technologies (ETT). 


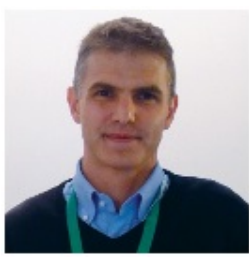

Alessandro Vanelli-Coralli (S'93-M'97-SM'07) received the Dr. Ing. Degree in Electronics Engineering and the Ph.D. in Electronics and Computer Science from the University of Bologna (Italy) in 1991 and 1996, respectively. In 1996, he joined the University of Bologna, where he is currently an Associate Professor. During 2003 and 2005, he was

a Visiting Scientist at Qualcomm Inc. (San Diego, CA), working in the Corporate R\&D Department on Mobile Communication Systems. Dr. Vanelli-Coralli chairs the PhD Board on Electronics, Telecommunications, and Information Technologies at the University of Bologna. Dr. Vanelli-Coralli has been Project Coordinator of the FP7 STREP CoRaSat (Cognitive Radio for SatCom), and Prime Investigator for several European Space Agency and European Commission funded projects. Dr. Vanelli-Coralli has been appointed member of the Editorial Board of the Wiley InterScience Journal on Satellite Communications and Networks, has been guest coEditor for several special issues in of international scientific journals, and has served as General Chairman and Technical Chairman of several scientific conferences. Dr. Vanelli-Coralli is co-recipient of several Best Paper Awards. 